\title{
Computer-assisted measurements of the histological structure of the tibial nerve and its terminal branches
}

\author{
Ł. Warchoł'1, J.A. Walocha1', E. Mizia'1, H. Liszka1', M. Bonczar², I. Zamojska' \\ 'Department of Anatomy, Jagiellonian University Medical College, Krakow, Poland \\ 2Intermed Medical Clinic, Zabierzow, Poland
}

[Received: 21 April 2020; Accepted: 7 June 2020]

Background: The aim of this study was to analyse the histological structure (cross-sectional area [CSA] and number of nerve bundles) of the distal part of the tibial nerve and its terminal branches (medial plantar nerve, lateral plantar nerve) using computer-assisted image analysis.

Materials and methods: The tibial nerve and its distal branches (medial and lateral plantar nerves) were dissected from the fresh cadavers. Each nerve was harvested $5 \mathrm{~mm}$ proximally and respectively $5 \mathrm{~mm}$ distally from the tibial nerve bifurcation, marked, dehydrated, embedded in paraffin, sectioned at $2 \mu \mathrm{m}$ slices and stained with haematoxylin and eosin. Then photographed and analysed using Olympus cellsens software.

Results: The studied group comprised 28 female and 32 male feet (mean age $68.1 \pm$ \pm 15.2 years). The mean CSA and the number of nerve bundles were respectively $17.86 \pm 4.57 \mathrm{~mm}^{2}, 33.88 \pm 6.31$ for the tibial nerve, $9.58 \pm 1.95 \mathrm{~mm}^{2}, 23.41 \pm$ \pm 7.37 for the medial plantar nerve and $7.17 \pm 2.36 \mathrm{~mm}^{2}, 15.06 \pm 5.81$ for the lateral plantar nerve in males and $12.27 \pm 2.45 \mathrm{~mm}^{2}, 26.32 \pm 8.87$ for the tibial nerve, $7.81 \pm 1.41 \mathrm{~mm}^{2}, 17.71 \pm 5.28$ for the medial plantar nerve and $5.83 \pm 1.25 \mathrm{~mm}^{2}$, $11.50 \pm 3.72$ for the lateral plantar nerve in females. Both CSA and number of nerve bundles of the tibial, medial plantar and lateral plantar nerves revealed no statistical differences when comparing foot side of the individual. The statistical difference was related to the gender, showing significantly bigger CSA and number of nerve bundles in males (CSA: $p=0.000, p=0.000, p=0.016$; number of nerve bundles: $p=0.01, p=0.003, p=0.004$, respectively). A positive correlation was found between the donor age and the tibial nerve CSA ( $r=0.44, p=0.000)$. A significant statistical difference was found between the medial and lateral plantar nerves both in CSA and number of nerve bundles ( $p<0.001, p<0.001$, respectively).

Conclusions: The CSA and the number of nerve bundles in the distal part of the tibial nerve and its branches are significantly larger in males with no differences between right and left foot of the individual. The tibial nerve shows increasing CSA with advanced age. The medial plantar nerve has larger CSA and more nerve bundles than the lateral plantar nerve. (Folia Morphol 2021; 80, 2: 372-379)

Key words: tibial nerve, cross-sectional area, nerve bundles, medial and lateral plantar nerves, computer-assisted image analysis, histology

Address for correspondence: Dr. Ł. Warchoł, Department of Anatomy, Jagiellonian University Medical College, ul. Kopernika 12, 31-034 Kraków, Poland, tel: +48 601865 239, e-mail: I.warchol@uj.edu.pl

This article is available in open access under Creative Common Attribution-Non-Commercial-No Derivatives 4.0 International (CC BY-NC-ND 4.0) license, allowing to download articles and share them with others as long as they credit the authors and the publisher, but without permission to change them in any way or use them commercially. 


\section{INTRODUCTION}

The tibial nerve is a peripheral sensorimotor nerve which is derived from the L4, L5 and S1-S3 spinal nerve roots [32]. It is the larger of the two terminal branches of the sciatic nerve arising in the popliteal fossa. It runs vertically on the tibialis posterior muscle together with the posterior tibial vessels. Postero-inferiorly to the medial malleolus it terminates emitting medial plantar nerve and lateral plantar nerve [26]. The tibial nerve bifurcation level shows a great variability with the most common occurrence below the tip of the medial malleolus, inside the tarsal tunnel [33].

Through its course the tibial nerve emits motor branches to the muscles of the posterior lower leg as well as sensory branches: medial sural cutaneous nerve and medial calcaneal nerve(s) innervating the skin of the posterolateral inferior third of the leg together with the lateral side of the foot and the skin of the heel, accordingly [10]. Medial calcaneal branches show diversity in terms of number (range from 1 to 4), location and nerve of origin [9, 17]. Both plantar nerves enter the sole of the foot supplying its muscles and skin. The medial plantar nerve innervates the skin medial to the line splitting fourth digit whilst the lateral plantar nerve the skin lateral to the line [20].

Tarsal tunnel syndrome is one of the entrapment conditions affecting the tibial nerve and its terminal branches in the medial ankle. It causes heel and sole burning pain and paraesthesia [2]. Such disorders together with other peripheral nerve pathologies may be examined by the ultrasound [24]. The cross-sectional area (CSA) is a parameter measured by the ultrasound which increasing value confirms the diagnosis [7].

The aim of this study was to assess the histological structure of the tibial nerve, medial plantar nerve and lateral plantar nerve as well as to determine the distribution of the nerve bundles of the distal tibial nerve to its terminal branches.

\section{MATERIALS AND METHODS}

The study was conducted on 60 lower limbs of the fresh cadavers in the Department of Anatomy of the Jagiellonian University Medical College between December 2016 and December 2019. The exclusion criteria were any deformation of the lower limb or the lower limb trauma, surgical or radiotherapeutic procedures of the lower limb, chronic disease of the lower limb in the medical record of the donor.

The research protocol was approved by the local Ethics Committee (registry no. 122.6120.315.2016).
The study has been performed in accordance with the ethical standards established in the 1964 Declaration of Helsinki and its later amendments.

\section{Dissection technique}

The incision was made in the midline between the tip of the medial malleolus and the Achilles tendon. It was continued $10 \mathrm{~cm}$ proximally along the Achilles tendon and $10 \mathrm{~cm}$ distally curving anteriorly $2 \mathrm{~cm}$ below the tip of the medial malleolus. Upon dissecting the skin and the subcutaneous tissue, the tibial nerve was visualised together with the posterior tibial artery and two posterior tibial veins. After meticulous dissection, the tibial nerve, its bifurcation and lateral and medial plantar nerves were exposed. The plantar nerves were marked $2 \mathrm{~cm}$ distally from the tibial nerve bifurcation point with the following pattern: blue thread - lateral plantar nerve, white thread - medial plantar nerve. The tibial nerve was left without any marking. Then $3 \mathrm{~cm}$ proximally to the bifurcation the tibial nerve was cut out from the main nerve trunk. Accordingly, $3 \mathrm{~cm}$ distally the medial and lateral plantar nerves were cut out. The excised tibial nerve and its terminal branches were removed en bloc from the cadaver. The incision was closed with the running subcuticular suture. The harvesting was carried out by the same surgeon.

\section{Preparation of histological slide}

The excised block of nerves was fixed in a $10 \%$ solution of the formaldehyde ( $\mathrm{pH} 7.4)$. After $2-5$ days it was removed from the formaldehyde. The tibial nerve was cut transverse to the nerve axis $5 \mathrm{~mm}$ and $10 \mathrm{~mm}$ proximally to the tibial nerve bifurcation point as were the medial and lateral plantar nerves $5 \mathrm{~mm}$ and $10 \mathrm{~mm}$ distally to the tibial nerve bifurcation point. Obtained $5 \mathrm{~mm}$ long nerve fragments were dehydrated separately and embedded in paraffin according to its initial marking. Each paraffin cube was transverse sectioned with the microtome providing one $2 \mu \mathrm{m}$ thick slice. Subsequently each slice was stained with haematoxylin and eosin (Fig. 1).

\section{Micromorphometry}

The CSA and the number of nerve bundles of the tibial nerve, the medial plantar nerve and the lateral plantar nerve were assessed using a light microscope (Olympus BX53, $20 \times$ magnification). Each cross-section was photographed (20 $\times$ magnification). Afterwards, the CSA was measured semi-automatically using Olympus cellSens Standard 2.3 software with the producer's 


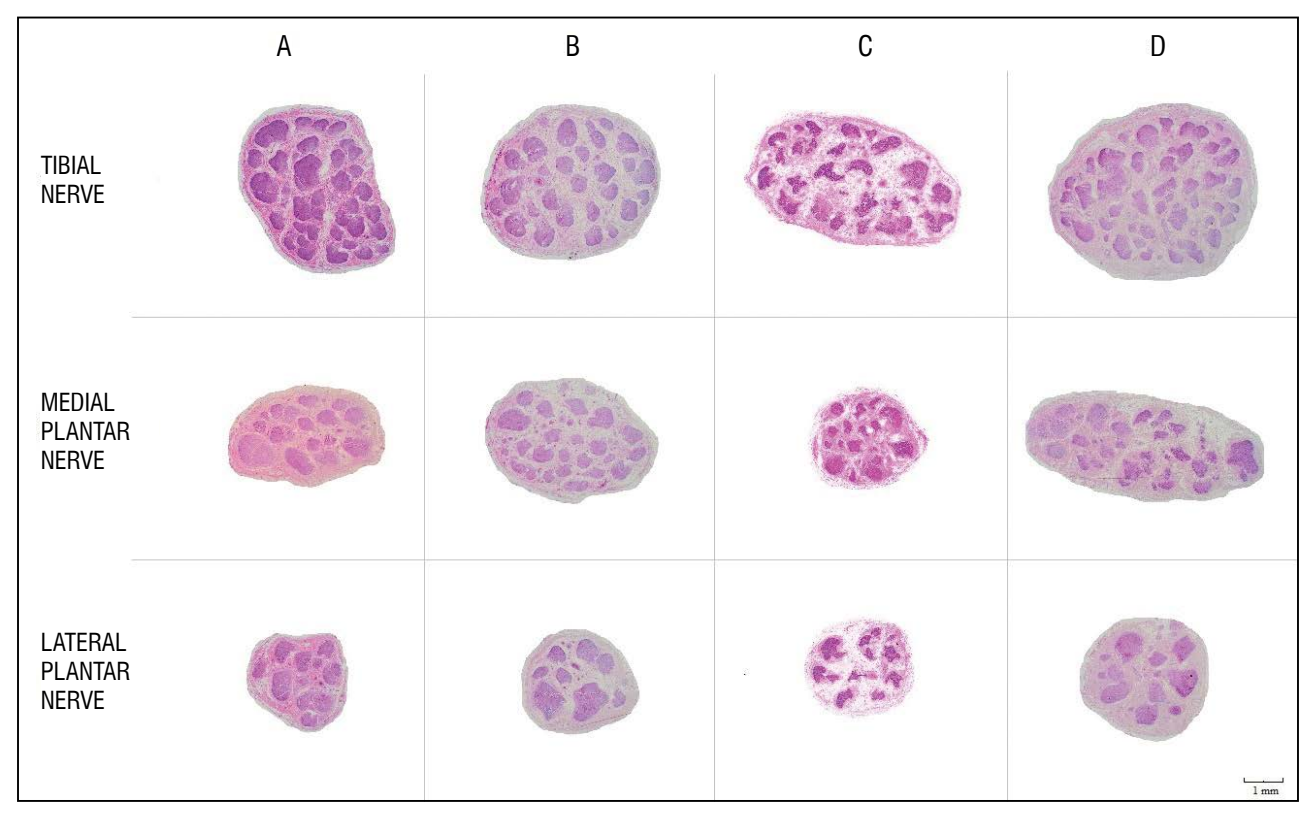

Figure 1. Cross-section of the tibial nerves (TN), the medial plantar nerves (MPN) and the lateral plantar nerves (LPN). Haematoxylin and eosin staining. $20 \times$ magnification; A. 58-year-old male, right food — cross-sectional area (CSA): TN $-12.76 \mathrm{~mm}^{2}, \mathrm{MPN}-6.79 \mathrm{~mm}^{2}$, LPN - $4.37 \mathrm{~mm}^{2}$; number of nerve bundles: TN $-35, \mathrm{MPN}-18, \mathrm{LPN}-12 ; \mathbf{B}$. 58-year-old male (same individual as in panel A), left food CSA: TN $-12.83 \mathrm{~mm}^{2}$, MPN $-9.92 \mathrm{~mm}^{2}$, LPN $-5.47 \mathrm{~mm}^{2}$; number of nerve bundles: TN - 29, MPN - 26, LPN - 8; C. 63-year-old male, right foot - CSA: TN $-13.92 \mathrm{~mm}^{2}$, MPN $-5.63 \mathrm{~mm}^{2}$, LPN $-5.05 \mathrm{~mm}^{2}$; number of nerve bundles: TN -34 , MPN -14 , LPN - 12; D. 84-year-old male, left food - CSA: TN $-18.39 \mathrm{~mm}^{2}$, MPN $-10.32 \mathrm{~mm}^{2}$, LPN $-7.09 \mathrm{~mm}^{2}$; number of nerve bundles: TN -44, MPN -34, LPN -13.

Table 1. The tibial nerve and its terminal branches measurements

\begin{tabular}{lllcccccc}
\hline Measurement & $\mathbf{N}$ & $\begin{array}{c}\text { Mean } \\
\text { I standard deviation }\end{array}$ & Median & Minimum & Maximum & $\begin{array}{c}\text { Lower } \\
\text { quartile (01) }\end{array}$ & $\begin{array}{c}\text { Upper } \\
\text { quartile (03) }\end{array}$ \\
\hline $\begin{array}{lllcccccc}\text { Cross-sectional } \\
\text { area }\left[\mathrm{mm}^{2}\right]\end{array}$ & Tibial nerve & 60 & $15.25 \pm 4.65$ & 14.66 & 7.22 & 30.82 & 11.77 & 17.29 \\
& Medial plantar nerve & 60 & $8.76 \pm 1.93$ & 8.45 & 5.53 & 14.22 & 7.19 & 9.90 \\
& Lateral plantar nerve & 60 & $6.54 \pm 2.02$ & 6.44 & 3.90 & 16.06 & 5.12 & 7.41 \\
Number of nerve & Tibial nerve & 60 & $30.35 \pm 8.45$ & 31.00 & 7.00 & 50.00 & 25.00 & 35.25 \\
bundles & Medial plantar nerve & 60 & $20.75 \pm 7.04$ & 20.00 & 5.00 & 38.00 & 16.00 & 25.00 \\
& Lateral plantar nerve & 60 & $13.40 \pm 5.22$ & 14.00 & 3.00 & 38.00 & 10.75 & 15.00 \\
\hline
\end{tabular}

precision of $10 \mu \mathrm{m}$, whilst the number of nerve bundles was calculated manually. Each slice was assessed once by the same pathologist. Then the values of the CSA and the number of nerve bundles were tabulated.

\section{Statistics}

Obtained data were statistically processed using descriptive statistics such as percentage, mean, median, standard deviation, upper and lower quartiles. A p-value of $<0.05$ was considered as statistically significant. Two groups were compared using the Mann-Whitney test and t-test for non-normally and normally distributed data, accordingly. Levene's test was used to check for homogeneity of variance. Two-way analysis of variance and possible interactions between the sex and age ( $>70 /<70$ years old) were checked in selected nerve parameters. Post-hoc analysis was performed using HSD test. To compare the nerve features between the left and right foot, the paired t-test or Wilcoxon rang test was used depending on whether data were normally or non-normally distributed. Correlation coefficients were calculated to establish any statistical dependence between parameters. All analyses were performed using MedCalc version 16.8 .

\section{RESULTS}

There were 30 fresh cadavers dissected ( $n=60$ lower limbs) with a mean age of $68.1 \pm 15.2$ (range from 27 to 91 years). 28 (46.7\%) feet were female and 
Table 2. The tibial nerve and its terminal branches measurements — comparison by gender

\begin{tabular}{|c|c|c|c|c|c|c|c|c|c|c|c|c|}
\hline \multicolumn{2}{|c|}{ Measurement } & \multicolumn{5}{|c|}{ Women } & \multicolumn{5}{|c|}{ Men } & \multirow[t]{2}{*}{$\mathbf{P}$} \\
\hline & & $\mathbf{N}$ & Mean \pm SD & Median & 01 & 03 & $\mathbf{N}$ & Mean \pm SD & Median & 01 & 03 & \\
\hline \multirow{3}{*}{$\begin{array}{l}\text { Cross-sec- } \\
\text { tional area } \\
{\left[\mathrm{mm}^{2}\right]}\end{array}$} & Tibial nerve & 28 & $12.27 \pm 2.45$ & 11.85 & 10.35 & 14.31 & 32 & $17.86 \pm 4.57$ & 17.10 & 15.02 & 19.90 & 0.000 \\
\hline & Medial plantar nerve & 28 & $7.81 \pm 1.41$ & 7.37 & 6.70 & 9.10 & 32 & $9.58 \pm 1.95$ & 9.16 & 8.40 & 10.66 & 0.000 \\
\hline & Lateral plantar nerve & 28 & $5.83 \pm 1.25$ & 5.77 & 4.61 & 6.86 & 32 & $7.17 \pm 2.36$ & 7.08 & 5.18 & 8.35 & 0.016 \\
\hline \multirow{3}{*}{$\begin{array}{l}\text { Number } \\
\text { of nerve } \\
\text { bundles }\end{array}$} & Tibial nerve & 28 & $26.32 \pm 8.87$ & 25.00 & 19.50 & 34.00 & 32 & $33.88 \pm 6.31$ & 34.00 & 28.50 & 38.00 & 0.001 \\
\hline & Medial plantar nerve & 28 & $17.71 \pm 5.28$ & 18.00 & 14.50 & 20.50 & 32 & $23.41 \pm 7.37$ & 23.00 & 17.50 & 29.50 & 0.003 \\
\hline & Lateral plantar nerve & 28 & $11.50 \pm 3.72$ & 12.00 & 9.00 & 14.00 & 32 & $15.06 \pm 5.81$ & 15.00 & 12.50 & 16.50 & 0.004 \\
\hline
\end{tabular}

Numbers in bold indicate statistically significant differences between males and females ( $<<0.05$ ); SD — standard deviation; 01 - lower quartile; 03 - upper quartile

Table 3. The tibial nerve and its terminal branches measurements - comparison by foot side

\begin{tabular}{|c|c|c|c|c|c|c|c|c|c|c|c|c|}
\hline \multicolumn{2}{|c|}{ Measurement } & \multicolumn{5}{|c|}{ Left foot } & \multicolumn{5}{|c|}{ Right foot } & \multirow[t]{2}{*}{$\mathbf{P}$} \\
\hline & & $\mathbf{N}$ & Mean \pm SD & Median & 01 & 03 & $\mathbf{N}$ & Mean \pm SD & Median & 01 & 03 & \\
\hline \multirow{3}{*}{$\begin{array}{l}\text { Cross-sec- } \\
\text { tional area } \\
{\left[\mathrm{mm}^{2}\right]}\end{array}$} & Tibial nerve & 30 & $15.82 \pm 5.08$ & 15.64 & 11.73 & 17.79 & 30 & $14.67 \pm 4.19$ & 14.14 & 11.91 & 16.64 & 0.229 \\
\hline & Medial plantar nerve & 30 & $8.80 \pm 1.98$ & 8.32 & 7.21 & 9.88 & 30 & $8.71 \pm 1.90$ & 8.78 & 7.08 & 9.92 & 0.805 \\
\hline & Lateral plantar nerve & 30 & $7.05 \pm 2.48$ & 6.99 & 5.05 & 8.30 & 30 & $6.03 \pm 1.28$ & 5.91 & 5.12 & 7.08 & 0.075 \\
\hline \multirow{3}{*}{$\begin{array}{l}\text { Number } \\
\text { of nerve } \\
\text { bundles }\end{array}$} & Tibial nerve & 30 & $30.43 \pm 8.36$ & 31.00 & 25.00 & 35.00 & 30 & $30.27 \pm 8.67$ & 32.00 & 24.00 & 37.00 & 0.989 \\
\hline & Medial plantar nerve & 30 & $20.37 \pm 6.77$ & 20.00 & 17.00 & 23.00 & 30 & 21.13 & 21.00 & 15.00 & 26.00 & 0.412 \\
\hline & Lateral plantar nerve & 30 & $14.20 \pm 6.37$ & 14.00 & 11.00 & 16.00 & 30 & $12.60 \pm 3.68$ & 13.00 & 10.00 & 15.00 & 0.296 \\
\hline
\end{tabular}

Statistically significant differences between left and right foot when $\mathrm{p}<0.05 ; \mathrm{SD}$ — standard deviation; 01 - lower quartile; 03 - upper quartile
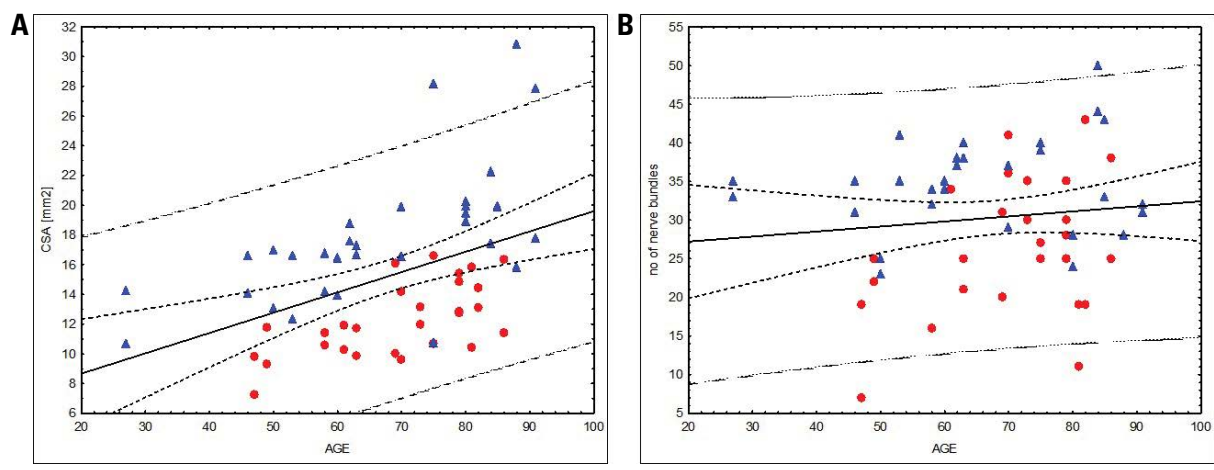

Figure 2. A. A scatter plot of donors age correlation with tibial nerve cross-sectional area (CSA). Triangles represent males $(r=0.69$, $p=0.000)$, dots represent females $(r=0.60, p=0.001)$; B. A scatter plot of donors age correlation with number of tibial nerve bundles. Triangles represent males $(r=-0.04, p=0.846)$, dots represent females $(r=0.31, p=0.110)$. The continuous line represents progression. The dash lines represent the $95 \%$ confidence intervals of the progression. The dash-dot lines represent the $95 \%$ prediction intervals.

32 (53.3\%) were male. The mean CSA and number of nerve bundles of the tibial nerve, the medial plantar nerve and the lateral plantar nerve are presented in Table 1. Differences between the gender and foot side are shown in Tables 2 and 3, respectively. Males showed larger CSA and more nerve bundles than females. No statistically significant differences between the right and left foot of the individual were found ( $p>0.05$ ). There was a statistically significant difference between medial and lateral plantar nerve both in CSA and number of nerve bundles ( $p<0.001$, $p<0.001$, respectively). The medial plantar nerve was confirmed to have 1.3 times larger CSA and 1.5 times more nerve bundles than the lateral plantar nerve. A positive correlation was noted between the age of donors and CSA of the tibial nerve $(r=0.44$, $p=0.000$ ) (Fig. 2). No statistically significant correlation was found between the age of donors and CSA 
Table 4. Studies of the tibial nerve cross-sectional area (CSA) measured at the level of medial malleolus

\begin{tabular}{|c|c|c|c|c|c|}
\hline & $\begin{array}{c}\text { Group } \\
\text { (n) }\end{array}$ & $\begin{array}{l}\text { Mean age } \\
\text { [years] }\end{array}$ & $\begin{array}{c}\text { CSA of the tibial nerve at the level } \\
\left.\text { of medial malleolus [ } \mathrm{mm}^{2}\right]\end{array}$ & $\begin{array}{c}\text { Reference range } \\
{\left[\mathrm{mm}^{2}\right]}\end{array}$ & Type of study \\
\hline He et al., 2019 [13] & 40 & 55.2 & $11.6 \pm 1.6$ & - & US 4-15 MHz \\
\hline Lothet et al., 2019 [24] & 15 & 21.7 & 12.3 & - & US $18 \mathrm{MHz}$ \\
\hline Singh et al., 2019 [30] & 45 & $30-68$ & $6.0 \pm 1.8$ & - & US 5-18 MHz \\
\hline Bedewi et al., 2018 [3] & 138 & 38.3 & $12.7 \pm 4.5$ & $2.0-30.0$ & US $18.5 \mathrm{MHz}$ \\
\hline Grimm et al., 2018 [12] & 100 & 51.2 & $10.2 \pm 2.0$ & - & US $14 \mathrm{MHz}$ \\
\hline Kronlage et al., 2017 [21] & 60 & 30.5 & $8.1 \pm 2.0^{*}$ & $4.0-12.1$ & MRI \\
\hline Singh et al., 2017 [29] & 75 & 39.5 & $12.4 \pm 1.1$ & $10.0-14.0$ & US 7-18 MHz \\
\hline Kang et al., 2016 [18] & 20 & 65.0 & $12.4 \pm 2.9$ & - & US 7-12 MHz \\
\hline Yiu et al., 2015 [35] & 29 & 11.3 & $6.3 \pm 1.9$ & $8.6-14.1$ & US 7-13 MHz \\
\hline Boehm et al., 2014 [5] & 56 & 50.2 & $9.6 \pm 2.2$ & $9.0-10.2$ & US $12-15 \mathrm{MHz}$ \\
\hline Seok et al., 2014 [28] & 94 & 43.9 & $12.1 \pm 3.1$ & $8.5-22.8$ & US 5-12 MHz \\
\hline Kerasnoudis et al., 2013 [19] & 75 & 53.5 & $6.3 \pm 1.5$ & $3.5-9.3$ & US $18 \mathrm{MHz}$ \\
\hline Riazi et al., 2012 [27] & 43 & 46.8 & $17.7 \pm 6.5$ & - & US 6-13 MHz \\
\hline Tagliafico et al., 2012 [31] & 58 & 47.0 & $9.6 \pm 4.0$ & $7.2-13.7$ & US $17.5 \mathrm{MHz}$ \\
\hline Cartwright et al., 2008 [7] & 60 & 45.9 & $13.7 \pm 4.3$ & $5.1-22.3$ & US $15 \mathrm{MHz}$ \\
\hline Ito et al., 2007 [16] & 35 & 52.8 & $7.9 \pm 1.5$ & 5.0-10.7 & US 7.5 MHz \\
\hline Lee et al., 2005 [22] & 24 & 57.4 & 12.0 & - & US 10-12 MHz \\
\hline
\end{tabular}

*Measured at the proximal third of the calf; US — ultrasonography; MRI — magnetic resonance imaging

of medial or lateral plantar nerves as well as number of nerve bundles. In the two-way analysis of variance, the mean CSA of the tibial nerve in males below 70 years of age was $15.37 \pm 0.80 \mathrm{~mm}^{2}$ and $20.35 \pm$ $\pm 0.80 \mathrm{~mm}^{2}$ for those above 70 years old, whilst in females $10.83 \pm 0.92 \mathrm{~mm}^{2}$ and $13.35 \pm 0.80 \mathrm{~mm}^{2}$, respectively. There was no statistically significant sex and age interactions with the CSA of the tibial nerve $(p=0.14)$. Post-hoc analysis revealed significant differences between younger and older males as well as between younger males and younger females $(p<0.05)$. Older males' CSA was significantly higher when compared to the younger and older females $(p<0.05)$.

\section{DISCUSSION}

The present study reveals data obtained using computer-assisted analysis of the histological structure of the distal tibial nerve and its terminal branches: medial and lateral plantar nerves. Literature analysis shows that the previous studies focused mostly on the variations of the topographic anatomy of the tibial nerve, its bifurcation, branching pattern and the cross-sectional area measured by the ultrasound imaging [33]. A limited number of studies concentrated on the histological structure of the tibial nerve and its distal branches. To the best of the authors' knowledge this is the first publication analysing histological structure of the medial and lateral plantar nerves as well as tibial nerve bundles distribution to its terminal branches. As the previous publications of the cross-sectional area were based on the ultrasound or magnetic resonance imagining, the present study is the first to reveal the CSA measured directly on the nerves harvested from the fresh cadavers, which shows greater accuracy.

In the present study, 60 lower limbs of the fresh cadavers were dissected. The majority of donors presented advanced age (mean age 68.1 years) and relatively equal gender distribution ( $53 \%$ males). The mean CSA of the tibial nerve measured with the computer-assisted image analysis is $15.25 \mathrm{~mm}^{2}$, which is comparable with the results obtained in the previous studies collected in Table 4. Nonetheless, it needs to be noticed that majority of those measurements are slightly below $15.25 \mathrm{~mm}^{2}$ as well as the mean age is lower than 68.1 years. It confirms the positive correlation between the age of the donors and the CSA observed in the present study. Despite different methodology (micromorphometry vs. ultrasound vs. magnetic resonance imaging) the obtained results showed insignificant differences in CSA of the tibial 
nerve. Thus, it proves the reliability and usefulness of those imagining methods.

Analysing the results summarised in Table 4, the authors found that the average cross-sectional area of the tibial nerve $\left(15.25 \mathrm{~mm}^{2}\right)$ is almost identical with Riazi et al. [27] $-17.7 \mathrm{~mm}^{2}$ and Cartwright et al. [6] $-13.7 \mathrm{~mm}^{2}$. At the same time it is more than two times larger than the values provided by Singh et al. [30] $-6.0 \mathrm{~mm}^{2}$, Yiu et al. [35] $-6.3 \mathrm{~mm}^{2}$ and Kerasnoudis et al. [19] $-6.3 \mathrm{~mm}^{2}$. The differences may be the result of the average age of the examined patients. Namely, Yiu et al. [35] examined children with the mean age of $11.3 \mathrm{~mm}^{2}$, which probably is the reason for the small CSA. The other cause of slight variation may be related to a different level of measurements. Lothet et al. [24] together with Kang et al. [18], Kerasnoudis et al. [19], Cartwright et al. [7], Bedewi et al. [3], Boehm et al. [5] and Grimm et al. [12] performed the examination at the level of the ankle whilst $\mathrm{He}$ et al. [13] and Singh et al. [30] measured the CSA $3 \mathrm{~cm}$ above the medial malleolus and Riazi et al. [27] $1 \mathrm{~cm}, 3 \mathrm{~cm}$ and $5 \mathrm{~cm}$ above the medial malleolus. As the tibial nerve bifurcation level shows great topographic variability, such inaccuracy may bring different results. Its location was subject of many studies and frequently referred to the medial or lower located lateral malleolus [23,34]. For the sake of comparison in the present study, all publications mentioned in Table 4 were qualified as if the measurements were at the level of medial malleolus. The other sources of the differences may be the reliability and accuracy of the researchers as well as the ultrasound resolution or the ethnical groups which was not comprised in the study.

To the best of the authors' knowledge, no previous studies of the cross-sectional area of the tibial, medial and lateral plantar nerves harvested from the fresh cadavers have been reported. The first report of the tibial nerve measurements dates back to 1938 when Horwitz [15] performed a dissection on 100 cadavers reporting the average diameter of tibial nerve to be between 6 and $10 \mathrm{~mm}$. Unfortunately, there is no information about the level of assessment. In 2006 Joshi and Joshi [17] examined 112 cadavers describing an average width of the tibial nerve above its bifurcation to be $8.23 \mathrm{~mm}$. The authors also measured the width of the medial plantar nerve to be $5.32 \mathrm{~mm}$ and the lateral plantar nerve to be $4.61 \mathrm{~mm}$. Since the tibial nerve shows clear flattening at the level of its bifurcation it would be wrong to assess the CSA using the circle area formula $\left(\pi \times r^{2}\right)$. These are the only measurements of the medial and lateral plantar nerves found in the literature. Complementing the available data with the mean CSA of $8.76 \mathrm{~mm}^{2}$ and the average number of nerve bundles to be 20.75 in the medial plantar nerve and $6.54 \mathrm{~mm}^{2}$ and 13.40 in the lateral plantar respectively should serve as a starting point for future researchers.

According to Alshami et al. [1], one of the causes of the foot pain is the tarsal tunnel syndrome. As the tibial nerve and its divisional branches pass through tarsal tunnel it may be entrapped or compressed. Joshi and Joshi [17] together with Bilge et al. [4] state that in the majority of cases the tibial nerve bifurcation is located inside the tarsal tunnel. As Heimkes et al. [14] point out, it is a tight, stretch resistant osteofibrous canal between talus, calcaneus and flexor retinaculum. It may be suggested that the larger the size (CSA) of the nerves (tibial, medial and lateral plantar), the higher the risk of its entrapment. Therefore the prevalence of the foot pain and paraesthesia among older people is higher.

Lothet et al. [24] together with Cartwright et al. [7] proved that in the medial ankle ultrasound examination the tibial nerve CSA remains uninfluenced by the patient's height and weight. In the present study the authors confirm that CSA of the tibial nerve increases with the advanced age, which is consistent with the findings of Grimm et al. [12] and Cartwright et al. [6]. It needs to be mentioned that according to Kerasnoudis et al. [19] and Mizia et al. [25] other peripheral nerves such as median nerve, radial nerve or sural nerve present an age-related decrease in the CSA values. This exceptional finding was explained by Ceballos et al. [8] in 1999 on the mouse model. The authors observed the age-related increase in mastocytes and macrophages depositing in the endoneurium as well as collagen accumulation in the perineurium, causing the enlargement of the cross-sectional area. Tibial nerve age-related thickening was also described by Grimm et al. [12] as a higher fibrous tissue deposition in the nerve.

In the present study, the number of nerve bundles in the tibial, medial plantar and lateral plantar nerves was also counted, finding respectively $30.35 \pm$ $\pm 8.45,20.75 \pm 7.04,13.40 \pm 5.22$ nerve bundles. Interestingly the number of nerve bundles of the tibial nerve is lower than the summative number of its two terminal branches (medial and lateral plantar nerves). The similar finding was reported by Delgado-Martinez 
et al. [11] who counted the number of nerve bundles of the median nerve. Despite the muscle and cutaneous branches sprouting from the main trunk of the median nerve along its course, the authors found the increasing number of nerve bundles in distal part of the forearm (11.81 \pm 0.32 in the proximal upper arm, $12.81 \pm 0.73$ in the distal upper arm, $21.87 \pm 0.58$ in the forearm). Although there is no study explaining this finding available in the literature, the authors suggest that the increased summative number of nerve bundles in the medial and lateral plantar nerves might result from the split of (some) nerve bundles of the tibial nerve at the bifurcation level. Therefore, finding out the branching pattern of the tibial nerve bundles and its distribution to the medial and lateral plantar nerves (by measuring the CSA of each nerve bundle or by counting the number of axons) at the bifurcation level might be an interesting subject for the future studies.

\section{Limitations of the study}

The fact that the cross-sectional area and number of nerve bundles were assessed on the nerves harvested from the fresh cadavers donated to the Department of the Anatomy results in the high average age of the examined group. Because of the technical difficulties no weight and height of the donors were obtained which might have been beneficial for this study. The other limitation of the present study is the fact that only one slice of each nerve was prepared for the micromorphometric assessment. Single pathologist, performing all measurements only once also biased the possibility to ascertain the inter-observer and intra-observer variabilities. Another restriction is a diverse level of the tibial nerve CSA measurements presented in available studies as well as lack of medial and lateral plantar nerve assessments which handicapped the comparison possibility.

\section{CONCLUSIONS}

To conclude, the authors of the present study proved that CSA and number of nerve bundles of the tibial nerve, medial plantar nerve and lateral plantar nerve are larger among males whilst shows no differences comparing to the side of the lower limb. This study also confirms that the CSA and number of nerve bundles of the medial plantar nerve is higher than the lateral plantar nerve. The authors proved the increasing CSA and number of nerve bundles among older donors. This work also contributes to the establishment of reference values for the medial and lateral plantar nerves.

\section{REFERENCES}

1. Alshami AM, Souvlis T, Coppieters MW. A review of plantar heel pain of neural origin: differential diagnosis and management. Man Ther. 2008; 13(2): 103-111, doi: 10.1016/j. math.2007.01.014, indexed in Pubmed: 17400020.

2. Bailie DS, Kelikian AS. Tarsal tunnel syndrome: diagnosis, surgical technique, and functional outcome. Foot Ankle Int. 1998; 19(2): 65-72, doi: 10.1177/107110079801900203, indexed in Pubmed: 9498577.

3. Bedewi MA, Abodonya A, Kotb M, et al. Estimation of ultrasound reference values for the lower limb peripheral nerves in adults: A cross-sectional study. Medicine (Baltimore). 2018; 97(12): e0179, doi: 10.1097/ MD.0000000000010179, indexed in Pubmed: 29561431.

4. Bilge $O$, Ozer MA, Govsa F. Neurovascular branching in the tarsal tunnel. Neuroanatomy. 2003; 2: 39-41.

5. Boehm J, Scheidl E, Bereczki D, et al. High-resolution ultrasonography of peripheral nerves: measurements on 14 nerve segments in 56 healthy subjects and reliability assessments. Ultraschall Med. 2014; 35(5): 459-467, doi: 10.1055/s-0033-1356385, indexed in Pubmed: 24764211.

6. Cartwright MS, Mayans DR, Gillson NA, et al. Nerve cross-sectional area in extremes of age. Muscle Nerve. 2013; 47(6): 890-893, doi: 10.1002/mus.23718, indexed in Pubmed: 23670837.

7. Cartwright MS, Passmore LV, Yoon JS, et al. Cross-sectional area reference values for nerve ultrasonography. Muscle Nerve. 2008; 37(5): 566-571, doi: 10.1002/mus.21009, indexed in Pubmed: 18351581.

8. Ceballos D, Cuadras J, Verdú E, et al. Morphometric and ultrastructural changes with ageing in mouse peripheral nerve. J Anat. 1999; 195 (Pt 4): 563-576, doi: 10.1046/j.1469-7580.1999.19540563.x, indexed in Pubmed: 10634695.

9. Davis TJ, Schon LC. Branches of the tibial nerve: anatomic variations. Foot Ankle Int. 1995; 16(1): 21-29, doi: 10.1177/107110079501600105, indexed in Pubmed: 7697149.

10. De Maeseneer $M$, Madani $H$, Lenchik $L$, et al. Normal anatomy and compression areas of nerves of the foot and ankle: US and MR imaging with anatomic correlation. Radiographics. 2015; 35(5): 1469-1482, doi: 10.1148/ rg.2015150028, indexed in Pubmed: 26284303.

11. Delgado-Martínez I, Badia J, Pascual-Font A, et al. Fascicular topography of the human median nerve for neuroprosthetic surgery. Front Neurosci. 2016; 10: 286, doi: 10.3389/ fnins.2016.00286, indexed in Pubmed: 27445660.

12. Grimm A, Axer $H$, Heiling B, et al. Nerve ultrasound normal values: Readjustment of the ultrasound pattern sum score UPSS. Clin Neurophysiol. 2018; 129(7): 1403-1409, doi: 10.1016/j.clinph.2018.03.036, indexed in Pubmed: 29729596

13. He $Y$, Xiang $X i$, Zhu BH, et al. Shear wave elastography evaluation of the median and tibial nerve in diabetic peripheral neuropathy. Quant Imaging Med Surg. 2019; 9(2): 273-282, doi: 10.21037/qims.2019.02.05, indexed in Pubmed: 30976551. 
14. Heimkes B, Posel P, Stotz S, et al. The proximal and distal tarsal tunnel syndromes. An anatomical study. Int Orthop. 1987; 11(3): 193-196, doi: 10.1007/BF00271447, indexed in Pubmed: 3623755.

15. Horwitz M . Normal anatomy and variations of the peripheral nerves of the leg and foot application in operations for vascular diseases: study of one hundred specimens. Arch Surg. 1938; 36(4): 626-636, doi: 10.1001/archsurg.1938.01190220068005.

16. Ito $T$, Kijima $M$, Watanabe $T$, et al. Ultrasonography of the tibial nerve in vasculitic neuropathy. Muscle Nerve. 2007; 35(3): 379-382, doi: 10.1002/mus.20673, indexed in Pubmed: 17058272.

17. Joshi SS, Joshi SD, Athavale AS. Anatomy of tarsal tunnel and its applied Significeance. J Anat Soc India. 2006; 55(1): 52-56

18. Kang S, Kim SeH, Yang SN, et al. Sonographic features of peripheral nerves at multiple sites in patients with diabetic polyneuropathy. J Diabetes Complications. 2016; 30(3): 518-523, doi: 10.1016/j.jdiacomp.2015.12.008, indexed in Pubmed: 26782023.

19. Kerasnoudis A, Pitarokoili K, Behrendt V, et al. Cross sectional area reference values for sonography of peripheral nerves and brachial plexus. Clin Neurophysiol. 2013; 124(9): 1881-1888, doi: 10.1016/j.clinph.2013.03.007, indexed in Pubmed: 23583024

20. Koo GB, Lee JH, Jang JH, et al. Superficial course of the medial plantar nerve: case report. Anat Cell Biol. 2019; 52(1): 87-89, doi: 10.5115/acb.2019.52.1.87, indexed in Pubmed: 30984458.

21. Kronlage $M$, Schwehr V, Schwarz D, et al. Magnetic resonance neurography: normal values and demographic determinants of nerve caliber and T2 relaxometry in 60 healthy individuals. Clin Neuroradiol. 2019; 29(1): 19-26, doi: 10.1007/s00062-017-0633-5, indexed in Pubmed: 29030674

22. Lee $D$, Dauphinée $D$. Morphological and functional changes in the diabetic peripheral nerve. J Am Podiatr Med Assoc. 2005; 95(5): 433-437, doi: 10.7547/0950433.

23. Lijoi F, Lughi M, Baccarani G. Posterior arthroscopic approach to the ankle: an anatomic study. Arthroscopy. 2003; 19(1): 62-67, doi: 10.1053/jars.2003.50003, indexed in Pubmed: 12522404.

24. Lothet EH, Bishop TJ, Walker FO, et al. Ultrasound-derived nerve cross-sectional area in extremes of height and weight. J Neuroimaging. 2019; 29(3): 406-409, doi: 10.1111/jon.12590, indexed in Pubmed: 30582247.

25. Mizia E, Tomaszewski KA, Rutowicz B, et al. Computer-assisted assessment of the histological structure of the human sural nerve. Folia Morphol. 2014; 73(3): 292-297, doi: 10.5603/FM.2014.0046, indexed in Pubmed: 25242156.

26. Moore KL. Clinically Oriented Anatomy. 8th Ed, LWW 2017.

27. Riazi S, Bril V, Perkins BA, et al. Can ultrasound of the tibial nerve detect diabetic peripheral neuropathy? A cross-sectional study. Diabetes Care. 2012; 35(12): 2575-2579, doi: 10.2337/dc12-0739, indexed in Pubmed: 23033242.

28. Seok HY, Jang JH, Won SJ, et al. Cross-sectional area reference values of nerves in the lower extremities using ultrasonography. Muscle Nerve. 2014; 50(4): 564-570, doi: 10.1002/mus.24209, indexed in Pubmed: 24639103.

29. Singh K, Gupta K, Kaur S. High resolution ultrasonography of the tibial nerve in diabetic peripheral neuropathy. J Ultrason. 2017; 17(71): 246-252, doi: 10.15557/ JoU.2017.0036, indexed in Pubmed: 29375899.

30. Singh Y, Dixit R, Singh S, et al. High resolution ultrasonography of peripheral nerves in diabetic peripheral neuropathy. Neurol India. 2019; 67(Suppl): S71-S76, doi: 10.4103/00283886.250719, indexed in Pubmed: 30688237.

31. Tagliafico A, Cadoni A, Fisci E, et al. Reliability of sideto-side ultrasound cross-sectional area measurements of lower extremity nerves in healthy subjects. Muscle Nerve. 2012; 46(5): 717-722, doi: 10.1002/mus.23417, indexed in Pubmed: 23055313.

32. Tomaszewski KA, Graves MJ, Henry BM, et al. Surgical anatomy of the sciatic nerve: A meta-analysis. J Orthop Res. 2016; 34(10): 1820-1827, doi: 10.1002/jor.23186, indexed in Pubmed: 26856540.

33. Warchol $€$, Walocha JA, Mizia E, et al. Ultrasound guided topographic anatomy of the medial calcaneal branches of the tibial nerve. Folia Morphol. 2020 [Epub ahead of print], doi: 10.5603/FM.a2020.0062, indexed in Pubmed: 32488855.

34. Warchoł Ł, Mróz I, Mizia E, et al. Vascular density of inferior tibiofibular joint: cadaveric experimental study. Folia Med Cracov. 2017; 57(1): 47-54, indexed in Pubmed: 28608862.

35. Yiu EM, Brockley CR, Lee KJ, et al. Peripheral nerve ultrasound in pediatric Charcot-Marie-Tooth disease type 1A. Neurology. 2015; 84(6): 569-574, doi: 10.1212/ WNL.0000000000001236, indexed in Pubmed: 25576636. 